\title{
Line Profile Variations of Rotating Pulsating Stars
}

\author{
C.Aerts \& M. De Pauw \\ Instituut voor Sterrenkunde, KULeuven, Belgium.
}

Line-profile variations (LPVs) are often seen in early-type stars. They were first detected in the $\beta$ Cephei stars, where they are due to radial as well as to nonradial pulsations (NRP). It is still a matter of debate whether NRP is also the cause of LPVs in broad-lined B- and Be stars.

The study of LPVs of nonradially pulsating stars is often considered to be especially interesting in the case of rapid rotators, because in such stars the line profiles may offer a Doppler image of the stellar surface. Almost all investigators conclude that only sectorial modes with high $\ell$-values can properly account for the observations of LPVs in broad-lined stars; on the other hand, high-degree modes are almost never seen in slowly rotating stars.

We point out here that the expression for the velocity field which is usually adopted, is not valid for rapid rotators. The Coriolis force implies that an individual pulsation mode should not be expressed in terms of a single spherical harmonic. We derived an expression for the components of the Lagrangian displacement field $\vec{\xi}$ which is exact to the first order in $\Omega / \omega$, where $\Omega$ is the rotation frequency and $\omega$ is the pulsation frequency. The approximation is valid in the case of $p$ - or low-order $g$-modes. Two toroidal correction terms, one with degree $\ell-1$ and one with degree $\ell+1$, and one spheroidal correction term with degree $\ell$, all having azimuthal number $m$, are found for $\vec{\xi}$ (where $\ell$ and $m$ are the degree and the azimuthal number of the mode in the non-rotating case).

From this result, we determined the velocity of a point on the stellar surface in the direction of the observer and studied the effect of the correction terms due to the Coriolis force on line profile variations. Theoretical profiles for $\ell=2$ and $\ell=4$ modes were calculated in the case of $\Omega / \omega=1,20,50 \%$ for an equatorial rotation velocity of $v_{\Omega}=200 \mathrm{~km} / \mathrm{s}\left(i=90^{\circ}\right)$ and a pulsation amplitude $v_{p}=20 \mathrm{~km} / \mathrm{s}$. In the case of axisymmetric modes, the Coriolis force has little effect on the line profiles. However, for tesseral and sectorial modes the line profiles are drastically affected by the correction terms, if $\Omega / \omega$ is of the order of $20 \%$ or larger, i.e. in the conditions which are met in broad-lined B stars and Be stars. It appears that "moving bumps" can then also occur for modes with low $\ell$-values (Aerts, in preparation).

The correction terms are so huge that they cannot be ignored if the aim is to obtain a reliable mode determination from line-profile fitting. In stars where the pulsation period is about equal to the rotation period it is probable that even the formalism presented here is not sufficient and that higher-order effects are important. 